\title{
The relationship between the level of development of social intelligence and the characteristics of pedagogical communication of young teachers
}

\author{
Oksana Elnikova ${ }^{1, *}$, Lyubov Komlik $^{1}$, Irina Faustova $^{1}$, Irina Filatova ${ }^{1}$ \\ ${ }^{1}$ Bunin Yelets State University. Yelets, Russia
}

\begin{abstract}
The article is devoted to the problem of social intelligence of young teachers, which is considered as the basis for the success of professional activities. The emphasis is put on studying the relationship between the level of development of social intelligence and the characteristics of pedagogical communication of a beginning teacher. The article presents the results of a pilot study that was carried out to identify a possible relationship between the mentioned parameters. The experiment involved young teachers (with no more than three years of work experience) of general educational institutions and final-year students of the university who had completed teaching practice. The level of development of social intelligence was studied with the help of the "Social Intelligence" test created by J. Guildford and M. Saliven (Russian adaptation by E.S. Mikhailova); the style of pedagogical communication was identified by the test called "Diagnostic methods of the styles of pedagogical communication" (authors: N.P. Fetiskin, V.V. Kozlov, G.M. Manuilov). The obtained data allows us for the conclusion that there is a relationship between the level of development of the social intelligence of beginning teachers and the style (model) of pedagogical communication used by them: teachers with more developed social intelligence use the most constructive and optimal models of pedagogical communication and interaction with students. The results of the research can be helpful in improving the quality of pedagogical education, professional training of students - teachers-to-be.
\end{abstract}

\section{Introduction}

In contemporary reality, psychological and pedagogical interest in the problem of social intelligence as one of the factors of effective communication and interpersonal interaction has increased. Today's transformations of the national educational system pose a challenge for the future teacher not only to continuously improve their knowledge and professional skills, but also to constantly expand the sphere of communication and interaction, to form skills and abilities in order to organize the process of pedagogical communication properly.

\footnotetext{
${ }^{*}$ Corresponding author: eln-oksana@yandex.ru
} 
On the back of information instability, the blurring of the boundaries of the communicative space and the strengthening of intercultural communication, active introduction of computer technologies into the educational process, more and more demands are made on the teacher, whether it is professional activity or the sphere of interpersonal relations. In this regard, the ability of a young teacher to be socially oriented, to successfully adapt to new working conditions, life tasks and difficulties, to interact with people around them in effective way - it all provides psychological well-being and success in teaching, and social intelligence acquires more importance.

Russian psychological science has got plenty of experience in studying a wide range of problems related to pedagogical communication, which imposes certain requirements on the personality and the organization of the process itself (Sh.A. Amonashvili, A.A. Bodalev, A.B. Dobrovich, A.P. Ershova, I.A. Zimnyaya, E.G. Zlobina, E.N.Ilyin, V.A.KanKalik, Ya.L. Kolominsky, M.Yu. Kondrat'ev, G.Yu. Ksenzova, S.Yu. Kurganov , A.A. Leontiev, O.V. Lesher, A.V. Mudrik, A.V. Petrovsky, I.I. Rydanova, Z.S. Smelkova, V.V.Shpalinsky and others).

Pedagogical communication is "a multidimensional process of organizing, establishing and developing communication, mutual understanding and interaction between teachers and students, generated by the goals and content of their joint activities" [12].

Pedagogical communication is one of the main tools of educating the younger generation. A.A. Bodalev pointed out that the results of the objective cognitive and practical activities of students depend on the organization of pedagogical communication.

According to N.V. Kuzmin, pedagogical communication is "communication between a teacher and schoolchildren in the learning process, which creates optimal conditions for the development of students' motivation and the creative nature of educational activities for the correct formation of the child's personality, providing a productive emotional climate for learning" [4].

I.A. Zimnyaya considers pedagogical communication "as a form of educational interaction and cooperation between teachers and students that simultaneously realizes communicative, perceptual and interactive functions and which uses the whole set of verbal, symbolic and kinetic means" [4].

From the L.D. Stolyarenko's point of view, pedagogical communication - "is a set of means and methods that ensure the implementation of the goals and objectives of education and training, which determine the nature of the interaction of the teacher and students" [14].

Most of modern scientists (M.P. Kulachenko, E.A. Ovsyannikova, L.M. Sharova etc.) note that the productivity of pedagogical activity is largely determined by the teacher's ability to organize communication with students in a proper way $[4,7,13,14]$.

From this perspective, on the basis of even a cursory analysis of the studied concept presented above, we can draw a conclusion that mutual understanding is achieved and interaction between teachers and students is built within the framework of pedagogical communication rather than anywhere else. Nevertheless, it should be noted that in practice, teachers and students often experience some difficulties in the emphasized area, and these difficulties are due to the inability or just low level of ability to understand another person, that is, the lack of development of social intelligence.

There are works in Russian psychology (E.S. Mikhailova, D.V. Ushakov, D.V. Lyusin, V.A.Genkina, N.A. Makarova, etc.) that testify to the relevance of the problem of social intelligence and the practical importance of research in this area.

"Social intelligence is the ability to understand another person, take into account the interests of other people, the ability to empathize, establish contacts, predict the development of communication processes, the ability to assess the social situation and correctly understand one's own role in it, understanding one's own rights and responsibilities, moral duty" [15]. 
According to modern science, social intelligence is the basis of productive personal socialization, successful social and psychological adaptation, effective communication and social interactions [7,10]. Social intelligence is the foundation for a professional development and the success of the performance of professional activities $[2,8,10,11]$.

In this regard, there is a need to raise the issue of the relationship between the social intelligence of the teacher and the style (model) of his pedagogical communication.

The relevance of the study of the role of the social intelligence of a teacher in pedagogical communication is determined by several circumstances:

- significant changes in the national educational system; expanding the boundaries of the information space; intensive introduction of computer technologies into educational work; large-scale development and introduction of distance technologies into the pedagogical process (which changes the system of building interaction);

- however, at the same time, a person remains a social being with an actual need for communication and interaction.

The teaching profession according to the classification proposed by E.A. Klimov, refers to the type of professions called "man-man" [5,6]. The characteristic feature of this type of profession is the paramount importance of communication and interaction with the social environment. Throughout the implementation of educational work by the means of pedagogical communication, the emphasis is made on the personal, intellectual, emotional spheres and the comprehensive development of the student [13].

The analysis of psychological and pedagogical literature and the experience of educational work made it possible to determine that one of the important factors of effective pedagogical communication is developed social intelligence. Thuswise, the aim of the study was to find the relationship between different levels of development of social intelligence with the preferred model of pedagogical communication of novice teachers.

Young teachers (with no more than three years of work experience) of general educational institutions of the Lipetsk region (300 examined people) and final-year students of the Bunin Yelets State University who had completed teaching practice (200 examined people) took part in the pilot study.

In order to obtain diagnostic data on the level of development of social intelligence, we used the test called "Social intelligence" created by J. Guildford and M. Sullivan (Russian adaptation by E.S. Mikhailova) [10]. In order to obtain diagnostic data on the style of pedagogical communication we used the "Methodology for diagnosing the styles of pedagogical communication" (authors are N.P. Fetiskin, V.V. Kozlov, G.M. Manuilov) [3].

The level of development of social intelligence was determined on the basis of a composite assessment (CA) obtained on the back of summing up the results of the performance of the examined people completed four subtests, reflecting the development of the cognitive ability and ability to predict human behavior [9].

The study of the styles of pedagogical communication by determining the leading model of interaction, thanks to the usage of the instruments mentioned above (methodology by N.P. Fetiskin, V.V. Kozlov, G.M. Manuilov), made it possible not only to identify the dominant style, but also to determine the level and nature of interaction between a teacher and a student, as well as to state a violation of this interaction. This, of course, is extremely important within the framework of the designated problem.

The results of the study revealed that only $7 \%$ of the surveyed have got mediumstrength social intelligence, these people are distinguished by a developed cognitive ability and ability to predict people's behavior in the course of interpersonal communication and interaction, effective communication, successful adaptation and professional activity. 49\% of teachers and senior students have got average social intelligence, therefore, the cognitive ability and ability to predict the behavior of people in communication and interaction is at an average level of development, they are distinguished by the ability to easily adapt to the 
surrounding reality and in their professional activity. $36 \%$ - medium-weak social intelligence, $8 \%$ - low social intelligence, these respondents have a poorly developed cognitive ability and ability to predict the behavior of people in the situation of interpersonal communication and interaction. They are common with the difficulties in communication and interactions and their ability to adapt in society is reduced.

In accordance with the obtained results, we have combined the sample group into two main groups: the first group included experimental subjects with medium-strong and medium social intelligence - 280 people; the second group - 220 people with medium-low and low social intelligence. The results are shown in Figure 1.

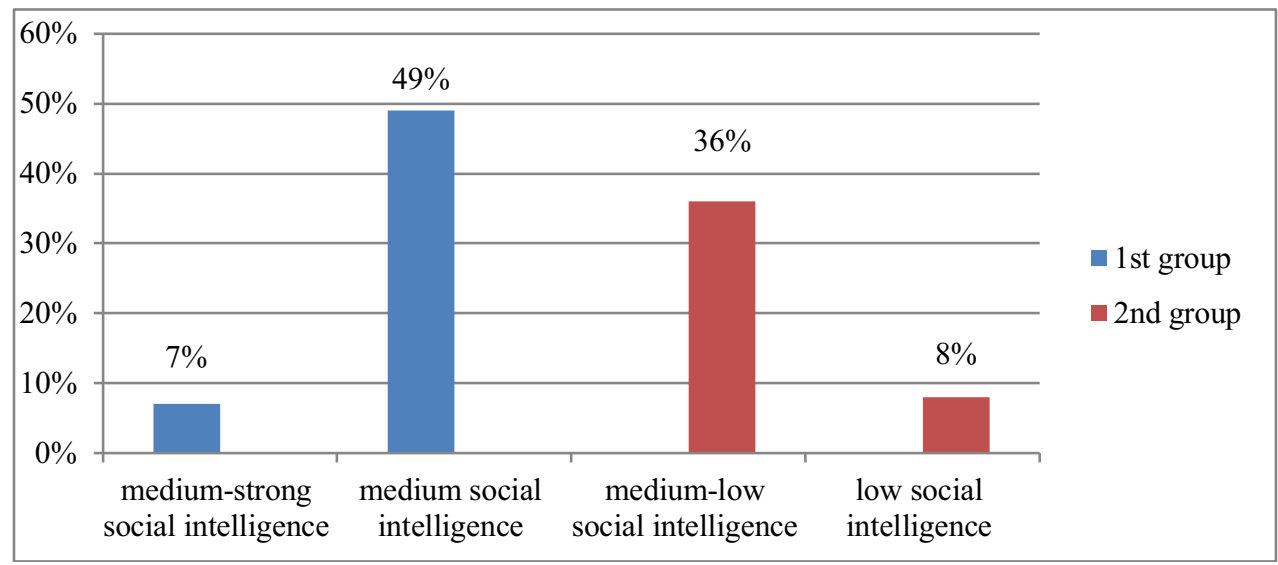

Fig. 1. Levels of development of social intelligence of young teachers according to the test of J. Guildford and M. Sullivan.

The style of pedagogical communication was derived from the model preference: noncontact ("China Wall"), hyporeflexive, hyperreflexive, differentiated attention, dictatorial, inflexible response, authoritarian, active interaction.

In the process of diagnosing of the pedagogical communication's styles, we received the following results (Figure 2).

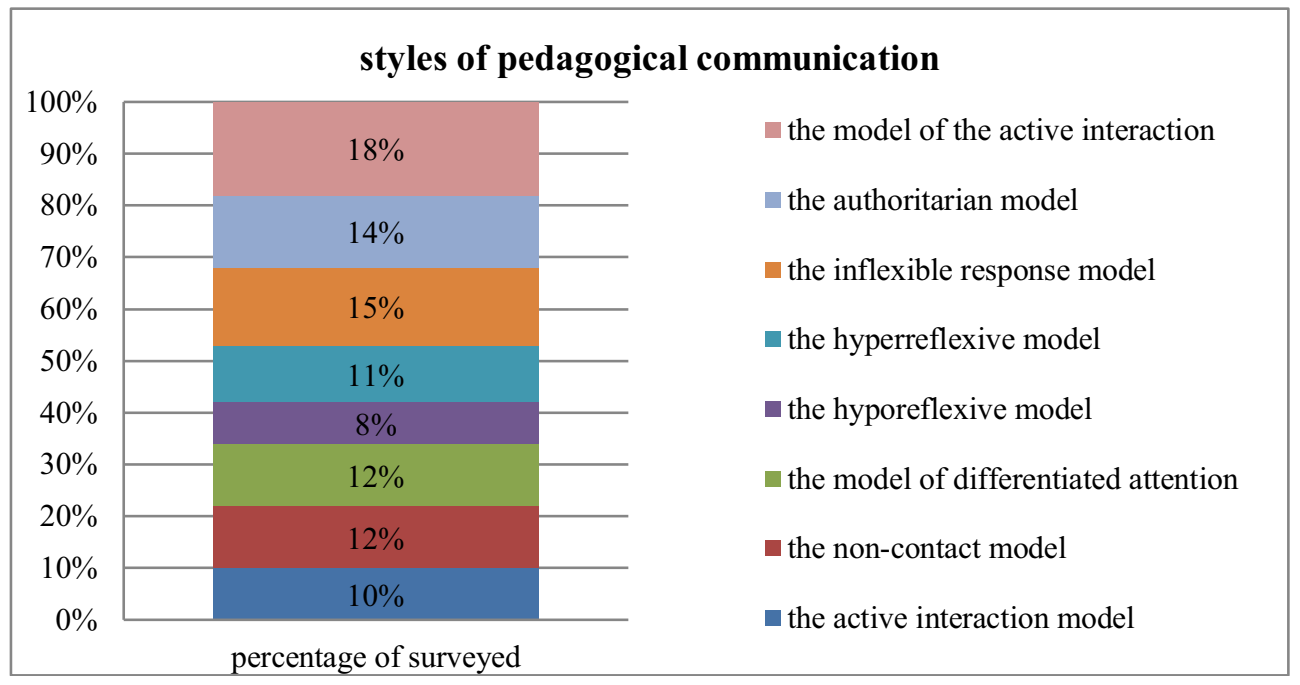

Fig. 2. Percentage ratio of styles of pedagogical communication according to the methodology by N.P.Fetiskin, V.V. Kozlov, G.M. Manuilov. 
We attributed $10 \%$ of the experimental subjects to the dictatorial model. These respondents do not show any personal interest in the release of information; in this case, we talk about the release of information without any interaction with the audience.

$12 \%$ demonstrated the non-contact model. It is characterized by the fact that against the background of the teacher's involuntary emphasis on his status and weak feedback between the teacher and the student, there is no desire for cooperation from the both sides, there is a predominance of the informational nature of the classes and a condescending attitude towards the students.

Another 12\% represented the model of differentiated attention, which is based on selective relationships with students. In terms of communication, such a teacher is focused not on the entire audience, but only on some part of it (excellent students, weak students, leaders or outsiders).

$8 \%$ of the subjects demonstrated the hyporeflexive model. In this case, the teachers' speech is in a form of a monologue, they seem to shut off themselves from the world, showing emotionlessness, while not reacting in any way to the children's group.

We referred $11 \%$ of the respondents to the hyperreflexive model, which expresses concerns about how the class perceives the teacher. Great importance is attached to interpersonal relations; there is an acute reaction to the psychological atmosphere in the students' environment. At the same time, there are constant nervousness and fear of being misunderstood.

$15 \%$ were diagnosed with the inflexible response model. Its characteristic feature reveals in the construction of the teacher's relationship with the class which is based on a rigid program without taking the age and ethnic characteristics of the students into account and there's deficiency of understanding any changes in the communication situation.

$14 \%$ demonstrated the authoritarian model. In this case, the teacher is at the center of the educational process, he asks questions and gives ready answers; the students' personal initiative is rejected. As a rule, there is minimal creative interaction during pedagogical communication.

$18 \%$ of the subjects referred to the model of the active interaction. Here, pedagogical communication is built in the form of an active dialogue with students, the initiative of children is encouraged, interaction prevails in a friendly manner, the role distance is preserved. Problems are solved together with children.

In order to confirm the statistical significance of the research results, we carried out mathematical processing of the obtained data using the Charles Spearman's correlation analysis [1]. We needed to find out whether there is a relationship between the level of development of a teacher's social intelligence and his preferred model of pedagogical communication (the data of the experimental study were processed in MicrosoftExel).

Based on the results of the correlation analysis, we can claim that there is a relationship: between medium-strong and strong social intelligence and such models of pedagogical communication as the model of differentiated attention (average 0.6), the hyperreflexive model (average 0.6), the inflexible response model (average 0,7 ), the active interaction model (high 0.9); between medium-low and low social intelligence and such models of pedagogical communication as the dictatorial model (average 0.6), the non-contact model (average 0.6), the hyporeflexive model (high 0.8), the authoritarian model (high 0.8). The research results are shown in Figure 3. 


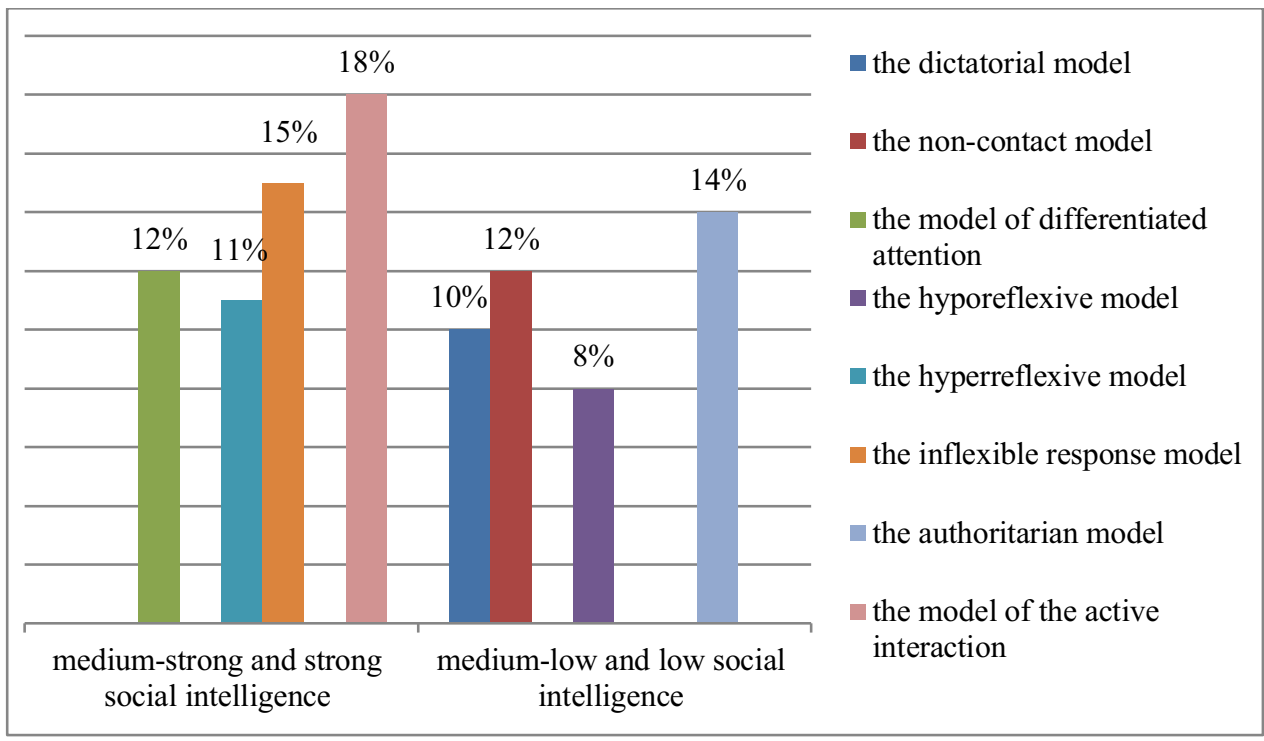

Fig. 3. Comparative results of diagnostics of the level of social intelligence and models of pedagogical communication.

Thus, based on the results of the pilot study, we can draw a conclusion that there is a relationship between the level of development of the social intelligence of novice teachers and the style (model) of pedagogical communication used by them: teachers with more developed social intelligence use the most constructive and optimal models of pedagogical communication and interaction with students.

Summing up the results of the work that we carried out, we can state that the problem of social intelligence is one of the recent psychological and pedagogical problems. It acquires particular importance today in the era of transformation of the educational system in Russia as well as in the world in the framework of settlement of a question of improving the quality of pedagogical education, professional training of students - future teachers, the development of effective psychological and pedagogical conditions and a model of the educational process in higher educational institutions.

The studies of the level of development of social intelligence among students mastering the teaching profession and its relationship with the characteristics of pedagogical communication are necessary both in theoretical and practical terms. This problem is significant for modern Russian education and for psychological and pedagogical science in general.

\section{References}

1. A. Büyül, P. Zefeldt, SPSS: The art of information processing. The analysis of statistical data and restoring hidden patterns (DiaSoftYup Publ., St. Petersburg, 2005) http://medinfo.krsu.edu.kg/images/SPSS.pdf

2. V.A. Genkina, The development of social intelligence of a teacher of a special school in professional practice, thesis....Cand. Sc. Education (Chelyabinsk State University, 2000)

3. N.P. Fetiskin, V.V. Kozlov, G.M. Manuilov, Diagnostics of styles of pedagogical communication (Publishing house of Institute of psychotherapy, Moscow, 2002) https://vsetesti.ru/146/ 
4. I.A. Zimnyaya, Educational psychology: Textbook for universities (Logos, M., 2005)

5. Yu.Ya. Golikov, Yu.P. Povarenkov, Yu.K. Strelkov et al, Psychological foundations of professional activity: chrestomathy (PERSE Publisher, Moscow, 2007) ISBN 978-59292-0165-3 http://www.iprbookshop.ru/7465.html

6. E.A. Klimov Psychology of professional self determination: textbook (Academy, Moscow, 2010)

7. V.N. Kunitsyna, Interpersonal communication ( Peter, SPb, 2001)

8. N.A. Makarova, Formation of social intelligence as a factor of self-realization of teachers: dissertation...Cand. Sc. Education (FSBEI of HPE "Transbaikal State University", 2013)

9. V.N. Mezinov, I.Yu. Filatova, Vestnik of Kostroma State University. Series: Pedagogy. Psychology. Sociokinetics 3, 136-141 (2019)

10. E.S. Mikhailova, Social intelligence: concepts, models, diagnostics (St. Petersburg University Publishing House, St. Petersburg, 2007) https://psytests.org/iq/guilford/guilford.html

11. Social Intelligence: Theories, Measurements, Research (Publishing house "Institute of Psychology RAS", M., 2004)

12. Pedagogy: Textbook for students of higher education. ped. study. institutions (M., 2004)

13. M.A. Slastenin, Pedagogy: textbook for students. Higher. study. Institutions (Publishing Center "Academy", M., 2007)

14. L.D. Stolyarenko, Pedagogical psychology (Phoenix, Rostov o / D, 2006)

15. O.B. Khlebodarova, Young scientist 11(34), $139-140$ https://moluch.ru/archive/34/3856/ 